\title{
Outcomes of patients with lymph node metastasis treated with radical prostatectomy and adjuvant androgen deprivation therapy in a Chinese population: results from a cohort study
}

\author{
Xiaojian Qin ${ }^{1,2+}$, Chengtao Han ${ }^{1,2+}{ }^{4}$, Hailiang Zhang ${ }^{1,2}$, Bo Dai ${ }^{1,2}$, Yao Zhu ${ }^{1,2}$, Yijun Shen ${ }^{1,2}$, Yiping Zhu ${ }^{1,2}$, \\ Guohai Shi ${ }^{1,2}$ and Dingwei $Y e^{1,2^{*}}$
}

\begin{abstract}
Background: The aim of this study is to assess the prognosis of prostate cancer ( $\mathrm{PCa}$ ) with lymph node metastases (LNM) detected in pelvic lymph node dissection (PLND) after radical prostatectomy (RP) and adjuvant androgen deprivation therapy (ADT) in a Chinese population.

Methods: From June 2005 to September 2012, the medical histories of 67 Chinese PCa patients with LNM detected after RP and extended PLND were collected, and all these patients received continuous adjuvant ADT. Postoperative survival was estimated using the Kaplan-Meier method. The impact of various clinicopathological factors on outcome was analyzed using Cox proportional hazard regression models. All tests were two-sided with $P<0.05$ considered significant.

Results: Median follow-up was 46.7 months, and two patients were lost to follow-up. Five-year event-free survival for patients with positive lymph nodes was 93.0\%, 83.0\%, and 96.0\% for local recurrence, systemic progression, and cancer death, respectively. One-year, 2-year, and 3-year biochemical recurrence (BCR)-free survival was 52\%, 40\%, and 22\%, respectively. Postoperative BCR-free survival was 25.7 months. BCR-free survival for patients with a single LNM was longer than those with two or more LNM (median 39.1 months vs. median 17.2 months, $P=0.002$ ). In a multivariate Cox model, only two or more LNM was a significant predictor of BCR (hazard ratio 2.6, $P=0.005$ ).
\end{abstract}

Conclusions: Despite low BCR-free survival, Chinese patients with LNM can benefit from RP and adjuvant ADT. Patients with low nodal metastatic burden had a favorable prognosis.

Keywords: Biochemical recurrence, Lymph node metastases, Pelvic lymph node dissection, Prostate cancer, Radical prostatectomy

\section{Background}

Prostate cancer ( $\mathrm{PCa})$ is the second most common cancer in men in the world. An estimated 1.1 million men worldwide were diagnosed with $\mathrm{PCa}$ in 2012, accounting for $15 \%$ of all cancers diagnosed in men. With an estimated 307,000 deaths in 2012, PCa was the fifth leading cause of death from cancer in men $(6.6 \%$ of total men deaths). In China, although PCa incidence and

\footnotetext{
* Correspondence: dwyeli@163.com

${ }^{\dagger}$ Equal contributors

'Department of Urology, Fudan University Shanghai Cancer Center, No.270 Dong'an Road, Shanghai 200032, China

${ }^{2}$ Department of Oncology, Shanghai Medical College, Fudan University, Shanghai, Shanghai 200032, China
}

C) Biomed Central

(c) 2015 Qin et al.; licensee BioMed Central. This is an Open Access article distributed under the terms of the Creative Commons Attribution License (http://creativecommons.org/licenses/by/4.0), which permits unrestricted use, distribution, and reproduction in any medium, provided the original work is properly credited. The Creative Commons Public Domain Dedication waiver (http://creativecommons.org/publicdomain/zero/1.0/) applies to the data made available in this article unless otherwise stated.

mortality rates remain low (age-standardized incidence and mortality rates, 5.3 per 100,000 and 2.5 per 100,000 , respectively in 2012), $\mathrm{PCa}$ is the most common and the most lethal male urogenital system cancer, as it is worldwide [1].

Patients who undergo staging scans prior to surgery and have no radiological evidence of pelvic lymphadenopathy, but are subsequently found to have involved pelvic lymph nodes at the time of pelvic lymph node dissection (PLND), have pathologically lymph node metastases (LNM) and have a favorable prognosis [2-4]. Patients with low-volume nodal metastases experience excellent survival rates, regardless of adjuvant treatment $[5,6]$. However, these 
studies were conducted in Western populations [2-6], and there is little data on the prognosis of PCa patients with LNM disease detected at the time of surgery in Chinese populations.

In such patients, Messing et al. from the Eastern Cooperative Oncology Group (ECOG) showed that early initiation of adjuvant androgen deprivation therapy (ADT) confers a survival benefit [7]; however, although a meta-analysis indicated benefit in prostate cancer-specific survival, no effect on overall mortality was identified for patients receiving adjuvant ADT, which resulted in a recommendation against adjuvant ADT [8]. Moreover, a cohort study carried out in the prostate-specific antigen (PSA) era found deferring immediate ADT in men with LNM after RP may not significantly compromise survival [9]. Regardless of variations among studies, adjuvant ADT is highly recommended for patients with LNM after radical prostatectomy (RP) worldwide range [10].

In this study, we assessed the prognosis in Chinese patients with LNM after RP and adjuvant ADT. Additionally, clinicopathological features were analyzed to identify predictors of disease progression.

\section{Methods}

We reviewed the medical records of 1,164 consecutive patients who underwent RP and extended PLND between June 2005 and September 2012 at our institute. The extension of PLND included not only the external and obturator regions as well as the portions medial and lateral to the internal iliac vessels but also the common iliac lymph nodes at least up to the ureteric crossing. Frozen section analyses of dissected lymph nodes were not routinely performed. In total, $67(5.8 \%)$ patients with LNM disease after surgery were identified and none of them received preoperative hormone therapy. Surgical procedures were performed by a single group of surgeons using standardized techniques. The 2002 primary tumor $(\mathrm{T})$, regional lymph nodes $(\mathrm{N})$, and distant metastases $(\mathrm{M})$ (TNM for short) classification system was used for staging, and the Gleason system was used for grading. All of the included patients with LNM disease received continuous adjuvant ADT within 90 days of RP.

Postoperative assessments, including physical examination and serum PSA measurements, were done monthly for the initial 2 years and quarterly thereafter. Radiographic evaluation was done as indicated clinically. Biochemical recurrence (BCR) was defined as PSA $0.2 \mathrm{ng} / \mathrm{mL}$ or greater. Local recurrence was defined as cancer on biopsy of the prostatic bed or imaging studies without evidence of systemic recurrence. Systemic progression involved demonstrable metastatic deposits on radionuclide bone scan or on biopsies other than biopsy of the prostatic bed. Patients with $\mathrm{BCR}$ or local recurrence were referred to palliative radiation. Cause of death was identified from death certificates or physician correspondence. In lieu of a formal ethics committee, the principles of the Helsinki Declaration were followed. All human subjects provided written informed consent with guarantees of confidentiality.

IBM IPSS statistics, version 20.0 (IBM Corp., Armonk, NY, US) was employed for statistical analyses. Postoperative survival was estimated using the Kaplan-Meier method with patients censored at last follow-up or death if the end point of interest was not attained. Predictors included PSA at diagnosis, Gleason score after RP, pathological stage (pT), as well as surgical margin status (SM) and number of LNM. The impact of various clinicopathological factors on outcome was analyzed using Cox proportional hazard regression models. The hazard ratios (HR) and 95\% confidence intervals (CI) associated with the presence and the number of LNM were estimated using the proportional hazards model. All tests were two-sided with $P<0.05$ considered significant.

\section{Results and discussion}

The clinical characteristics of the 67 patients with LNM after surgery are summarized in Table 1 . At a median follow-up of 46.7 months (range, 24 to 110 months), two patients were lost to follow-up, 43 patients had BCR, five experienced local recurrence, seven had systemic relapse, and four died, including two from PCa. Five-year event-free survival for patients with positive lymph nodes was $93.0 \%$, $83.0 \%$, and $96.0 \%$ for local recurrence, systemic progression, and cancer death, respectively. Since the volume of patients with local recurrence, systemic progression, and cancer death were limited, only BCR-free survival was further analyzed, and Figure 1 shows the Kaplan-Meier survival curves for patients with LNM disease. One-year, 2-year, and 3-year BCR-free survivals were $52 \%, 40 \%$, and $22 \%$, respectively.

\section{Table 1 Patient characteristics}

\begin{tabular}{ll}
\hline Characteristics & Patients, $\boldsymbol{n}=\mathbf{6 7}$ \\
\hline Age at surgery, year ,median (IQR) & $67(63 \sim 71)$ \\
Preoperative PSA, ng/ml & $46.0(19.3 \sim 94.6)$ \\
Positive surgical margin, no. (\%) & $12(10 \sim 18)$ \\
Nodes removed, no., median (IQR) & \\
Positive nodes removed, no. (\%) & \\
1 & $37(55.2)$ \\
2 & $16(23.9)$ \\
$\geq=3$ & $14(20.9)$ \\
Pathologic Gleason grade, no. (\%) & \\
$\quad \leq=7$ & $22(32.8)$ \\
$>7$ & $45(67.2)$ \\
Tumor stage, no. (\%) & \\
$\quad \leq=$ T2 & $19(28.4)$ \\
$>2$ & $48(71.6)$ \\
\hline
\end{tabular}

$\mathrm{IQR}=$ interquartile range; $\mathrm{PSA}=$ prostate-specific antigen . 


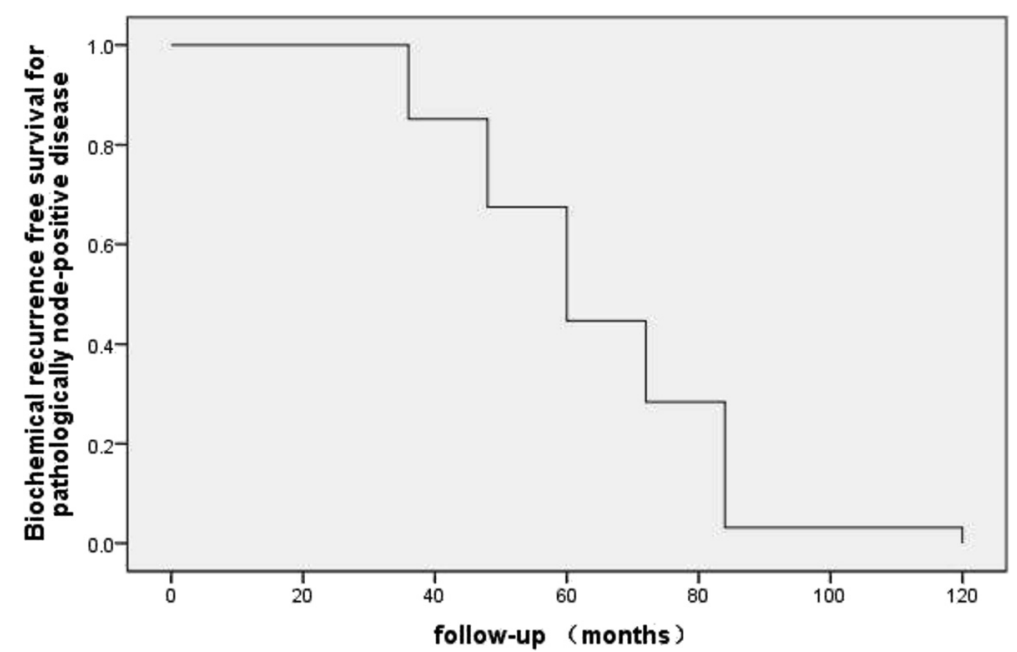

Figure 1 Biochemical-free survival of patients with lymph nodes metastases disease after radical prostatectomy and adjuvant androgen deprivation therapy.

Postoperative BCR-free survival was 25.7 months and was then stratified for all LNM patients who underwent RP by the number of positive lymph nodes (Figure 2). The BCR-free survival for patients with a single LNM was longer than that for patients with two or more LNM (median 39.1 months, 95\% CI, 24.7 to 53.5, vs. median 17.2 months, $95 \%$ CI, 13.7 to $20.7 ; P=0.002$ ). Although two metastatic nodes significantly increased the risk of BCR (HR 2.7, 95\% CI 1.3 to 5.5; $P=0.008$ ), increasing nodal involvement did not worsen patient risk of BCR (HR 1.1, 95\% CI 0.5 to 2.6; $P=0.797$ ). One-year, 2-year, and 3-year BCR-free survival for patients with a single LNM and two or more LNM were $69 \%$ vs. $31 \%, 58 \%$ vs. $18 \%$, and $28 \%$ vs. $18 \%$, respectively.

We next examined risk factors for disease progression in patients with positive lymph nodes. In a multivariate Cox model, only two or more LNM was a significant predictor of BCR (Table 2).

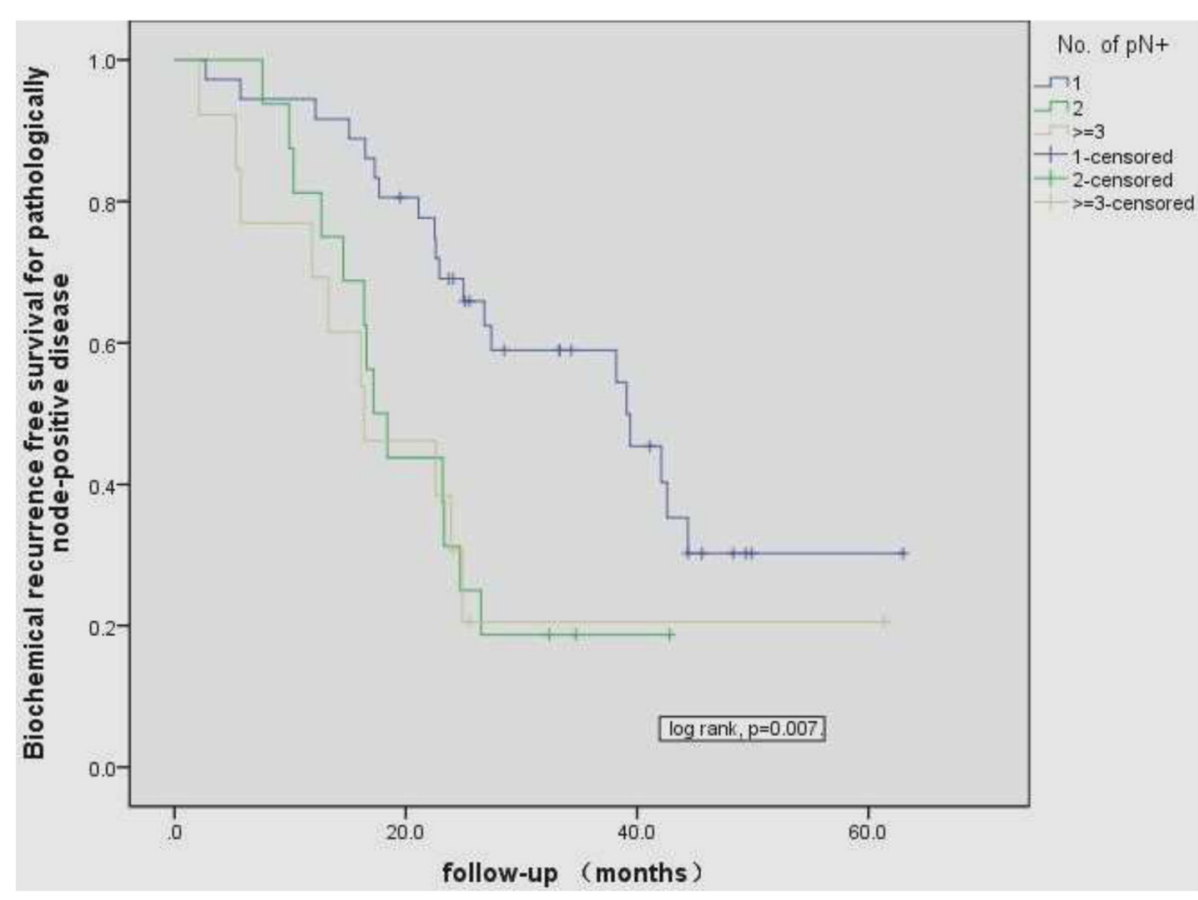

Figure 2 Biochemical-free survival of patients with different numbers of positive lymph nodes after radical prostatectomy and adjuvant androgen deprivation therapy. 
Table 2 Multivariate analysis of risk factors associated with biochemical recurrence from prostate cancer after radical prostatectomy in patients with node-positive disease

\begin{tabular}{lcc}
\hline Risk factor & HR $(\mathbf{9 5 \%} \mathbf{C l})$ & $\begin{array}{l}\boldsymbol{P} \text { value } \\
\text { (chi-square test) }\end{array}$ \\
\hline Preoperative PSA (>=20 ng/ml or not) & $0.6(0.3$ to 1.4$)$ & 0.246 \\
Pathologic Gleason grade ( $>7$ vs. $\leq=7)$ & $1.1(0.6$ to 2.1$)$ & 0.795 \\
Tumor stage (pT3/4 vs. pT2) & $1.4(0.6$ to 3.0$)$ & 0.435 \\
Positive surgical margin & $0.9(0.5$ to 1.9$)$ & 0.853 \\
Positive nodes removed & $2.6(1.3$ to 5.1$)$ & 0.005 \\
(2 or more vs. 1) & & \\
\hline
\end{tabular}

$\mathrm{HR}=$ hazard ratio; $\mathrm{Cl}$ = confidence interval; $\mathrm{PSA}=$ prostate-specific antigen.

A larger Munich Cancer Registry series reported that the incidence of nodal metastases in a European population between 1988 and 2007 was generally 10.2\% during PLND and completed or abandoned RP [11]. Between the 1980s and early 2000s, nodal metastases in US patients with presumed clinically localized PCa decreased by $4 \%$ to $6 \%$, likely because of the earlier detection of cancer with PSA screening [12-14]. The Mayo series revealed a marked decline in the incidence of positive lymph nodes at the time of RP, from $9.1 \%$ between 1988 and 1993 to $1.8 \%$ between 1998 and 2001 [2]. A small cohort of Chinese patients in Taiwan had a reported rate of LNM disease of 5.6\% from 1993 to 2001 [15]. In our cohort, we experienced a similar rate $(5.8 \%)$ of LNM detected after RP in Chinese patients. Presently, there is no consensus on either the treatment or prognosis in these Chinese patients.

The advent of lymph node metastasis marks the transition from localized to systemic disease. It is a generally accepted concept that disseminated malignant disease requires systemic treatment. Regardless of this paradigm, the issue of whether RP is justified in patients with limited lymph node involvement remains controversial. Because of PSA-based earlier detection of metastasis, the incidence of patients presenting with lymph node metastasis at the time of diagnosis has markedly decreased, approaching only $5 \%$ at larger institutions. Therefore, it is unlikely that a prospective randomized trial to address this issue will ever be successfully performed, thereby rendering data from carefully conducted retrospective studies important.

The Mayo series included 507 lymph node-positive patients who received RP at the time of PLND, with the longest follow-up being 10.3 years, revealed a 10-year prostate cancer-specific survival (PCSS) of $86 \%$, with $56 \%$ of men free of BCR at the time of their last follow-up visit. In this series, $90 \%$ of the patients received adjuvant $\mathrm{ADT}$ and 9\% received adjuvant RT. On multivariate analysis, Gleason score 8 to 10, positive surgical margins, and two or more positive lymph nodes were adverse predictors of PCSS, whereas adjuvant ADT use was not associated with systemic progression or cancer-specific survival [2]. In the Munich Cancer Registry series, 688 patients were similarly treated with RP after PLND revealed LNM disease, whereas 250 were treated without RP. About 75\% of all patients received adjuvant ADT and 25\% received adjuvant RT, with adjuvant therapies equally balanced between the two groups. With a median follow-up of 5.6 years, overall survival was significantly better in the RP group, and after adjustment for age, clinical $\mathrm{T}$ stage, number of LNM, tumor grade, and PSA level, patients who underwent RP were more likely to be alive; however, the cutoff point of two or more positive LNs reported in the Mayo series was not reproduced with their data [11].

Other series' data have reported the prognosis of LNM disease without adjuvant ADT [3-5,16] and have come to a consensus that patients with low-volume nodal metastases experience excellent survival rates. Despite the idea that patients with LNM disease after RP have a favorable prognosis, what is not consistent among studies is how many positive nodes detected during RP are an indicator of poor prognosis $[2-5,11,16]$. In the present cohort with adjuvant ADT, we found the BCR-free survival for patients with a single positive node was longer than that for patients with two or more positive nodes. Although two metastatic nodes significantly increased the risk of $\mathrm{BCR}$, increasing nodal involvement did not worsen the patient risk. Clinicians should be alerted to the fact that the prognosis is not good for patients with two or more positive lymph nodes after RP in Chinese patients, even with adjuvant ADT. Our data seems consistent with some previous series' $[2,5,16]$.

It is alarming that 1-, 2-, and 3-year BCR-free survival rates were $52 \%, 40 \%$, and $22 \%$ for these patients with adjuvant ADT. Moreover, the 1-, 2-, and 3-year BCR-free survival for patients with a single positive node and two or more positive nodes were $69 \%$ vs. $31 \%, 58 \%$ vs. $18 \%$, and 28 vs. $18 \%$, respectively, indicating that, even for PCa patients with limited LNM, the combination of RP, extended PLND, and adjuvant ADT hardly provided a cure, and around $80 \%$ of these patients might be faced with BCR within 3 years after RP. Von Bodman et al. reported a 2 -year BCR-free survival rate of $55 \%$ in a US cohort without adjuvant therapy [5]; with adjuvant ADT, Boorjian et al.'s US cohort had a 5-year BCR-free survival of $69 \%$ [2]; without adjuvant therapy, the 5-year BCR-free survival from studies by Touijer et al. and Bader et al. were $35 \%$ and $82 \%$, respectively $[4,16]$.

Moreover, there is no consensus on predictors of disease progression. Only specimen Gleason score and the number of positive lymph nodes were independent predictors of BCR in von Bodman's series [5]. The same association was reproduced in a retrospective study by Touijer et al., which investigated the long-term outcomes of patients with LNM treated with RP without adjuvant ADT [4]. In 
a multivariate Cox model, only increased preoperative PSA and nondiploid tumor ploidy were significant predictors of BCR [2]; in a multivariate Cox proportional hazards model including the number of lymph node metastases, tumor stage, and Gleason score, the number of positive nodes was the only variable affecting progression and cancer-specific death [16]. In our multivariate Cox model, only two or more LNM was a significant predictor of BCR, with a hazard ratio of 2.6, compared with those with one single positive node.

We should acknowledge data of a previous Chinese cohort, in which they reported excellent prognosis following LNM disease in a single Chinese institution [17]. With laparoscopic radical prostatectomy (LRP), extended PLND (including external iliac artery, common iliac artery, obturator fossa, internal iliac, and presacral lymph nodes), and 9 months' adjuvant ADT, 3-year BCR-free survival for their 47 LNM PCa patients was reported to be $59.2 \%$. Additionally, they found in their cohort that there were no significant differences in BCR-free overall and cancer-specific survival rates between lymph node-positive and lymph node-negative PCa. Compared with their data, our cohort in Chinese patients failed to provide the same optimistic results.

Bader et al. reported that meticulous PLND with no adjuvant therapy produced intriguing results. The authors described dissection of tissues along the external iliac vein, in the obturator fossa, and along the internal iliac artery, with removal of a median of 21 lymph nodes. The projected probabilities of overall survival and PCSS for these 88 patients were both $74 \%$ at 5 years [16]. Meticulous pelvic lymph node dissection, particularly in patients with micrometastases, seems not only to be a staging procedure but may also have a positive impact on disease progression and long-term disease-free survival [16]. Compared with a median 12 lymph nodes in PLND, in the other Chinese cohort, median 19.5 lymph nodes were removed, including those from the presacral area, and this might contribute to a better prognosis in BCR-free survival [17].

However, the prognosis of Chinese patients in our cohort seemed acceptable when we considered the 5-year survival. Although the volume of patients with local recurrence, systemic progression, and cancer death were quite limited in the present cohort, the 5-year event-free survival for patients with positive lymph nodes was $93.0 \%, 83.0 \%$, and $96.0 \%$ for local recurrence, systemic progression, and cancer death, respectively, which are encouraging. In a previous retrospective analysis performed in 431 consecutive patients treated for $\mathrm{PCa}$ at six Chinese institutions, they found that the management of $\mathrm{PCa}$ in China differs from that in Western countries, and the median BCR-free survival in patients with advanced diseases were 13 to 14.1 months after surgical or medical ADT [18]. Compared with their data, the prognosis of patients with LNM PCa in our cohort seemed better with a median BCR-free survival of 25.7 months. We think this study added to the body of evidence that cytoreduction of the primary tumor allows a better response in advanced $\mathrm{PCa}$ to androgen ablation, as was suggested by our own experience in metastatic hormone-sensitive prostate cancer (mHSPC). In our previous cohort of mHSPC patients, transurethral resection of the prostate resulted in better and more prolonged response to hormone therapy, with a trend toward positive influence in disease-specific survival and overall survival [19]. It seemed that low BCR-free survival in the present cohort was not necessarily indicative of poor prognosis in a Chinese population with LNM.

Earlier, we proposed a hypothesis that one possible mechanism that underlies the increased response to systemic therapy in advanced prostate cancer after local surgery on the primary tumor is the different androgen microenvironment inside and outside the prostate [19]. Previous investigations have found that medical castration reduces tissue androgens by $75 \%$ and also reduces the expression of several androgen-regulated genes. However, many androgen-response genes, including the androgen receptor and PSA, are not suppressed after short-term castration or after 9 months of neoadjuvant ADT. The degree of medical castration based on serum testosterone levels cannot be equated with the thoroughness of androgen ablation in the prostate microenvironment. Standard androgen deprivation does not consistently suppress androgen-dependent gene expression because of higher levels of intraprostatic androgens. Suboptimal suppression of tumoral androgen activity may lead to adaptive cellular changes, allowing prostate cancer cell survival in a low-androgen environment [20]. Because the primary tumor might be the primordial source of metastatic disease and because newly disseminated cancer cells from the castrated prostate are probably more hormone refractory, reducing the volume of intraprostatic cancer cells by local surgery before they are castration adaptive may reduce the proportion of hormone refractory cells disseminated later [21]. This idea is in accordance with what was found in the present cohort and others, as patients with low-volume nodal metastases experience better survival with RP and adjuvant ADT [2-4].

The present study has several limitations. For instance, the follow-up was not long enough to include sufficient events of local recurrence, systemic progression, or cancer death; therefore, the analysis was limited to BCR, which may restrict its immediate application for long-term survival. Although our study represents a retrospective, single center experience, the decreasing incidence of lymph node-positive prostate cancer during the PSA era makes future prospective, randomized trials of these patients unlikely. There are little data on the prognosis of 
PCa patients with LNM disease detected at the time of surgery in Chinese populations. As a single center cohort report, our data provide primary experience in the management of such patients, but further multicenter studies in Chinese populations are obviously needed for validation.

\section{Conclusions}

Despite low BCR-free survival, Chinese patients with LNM can benefit from RP and adjuvant ADT. Patients with low nodal metastatic burden have a favorable prognosis. We think this study also adds to the evidence that cytoreduction of the primary tumor allows a better response of advanced PCa to androgen ablation.



ADT: androgen deprivation therapy; BCR: biochemical recurrence; $\mathrm{Cl}$ : confidence intervals; HR: hazard ratios; LNM: lymph node metastases; PCa: prostate cancer; PLND: pelvic lymph node dissection; PSA: prostate-specific antigen; pT: pathological stage; RP: radical prostatectomy; SM: surgical margin status; TNM: primary tumor regional lymph nodes and distant metastases.

\section{Competing interests}

All the authors have agreed to its submission. The authors declare no conflicts of interest.

\section{Authors' contributions}

$\mathrm{XJQ}, \mathrm{CTH}$, and DWY designed the study. XJQ and CTH drafted the manuscript. HLZ, BD, YZ, YJS, YPZ, and GHS provided the clinical data of patients who underwent radical prostatectomy. All authors read and approved the final manuscript.

\section{Acknowledgments}

This research was not supported by any funds.

Received: 4 January 2015 Accepted: 24 April 2015

Published online: 06 May 2015

\section{References}

1. Ferlay J, Soerjomataram I, Dikshit R, Eser S, Mathers C, Rebelo M, et al. Cancer incidence and mortality worldwide: sources, methods and major patterns in GLOBOCAN 2012. Int J Cancer. 2015;136:E359-86.

2. Boorjian SA, Thompson RH, Siddiqui S, Bagniewski S, Bergstralh EJ, Karnes RJ, et al. Long-term outcome after radical prostatectomy for patients with lymph node positive prostate cancer in the prostate specific antigen era. J Urol. 2007;178:864-70. discussion 870-861.

3. Schumacher MC, Burkhard FC, Thalmann GN, Fleischmann A, Studer UE. Good outcome for patients with few lymph node metastases after radical retropubic prostatectomy. Eur Urol. 2008;54:344-52.

4. Touijer KA, Mazzola CR, Sjoberg DD, Scardino PT, Eastham JA. Long-term outcomes of patients with lymph node metastasis treated with radical prostatectomy without adjuvant androgen-deprivation therapy. Eur Urol. 2014;65:20-5.

5. von Bodman C, Godoy G, Chade DC, Cronin A, Tafe LJ, Fine SW, et al. Predicting biochemical recurrence-free survival for patients with positive pelvic lymph nodes at radical prostatectomy. Journal Of Urology. 2010;184:143-8.

6. Briganti A, Blute ML, Eastham JH, Graefen M, Heidenreich A, Karnes JR, et al. Pelvic lymph node dissection in prostate cancer. Eur Urol. 2009:55:1251-65.

7. Messing EM, Manola J, Yao J, Kiernan M, Crawford D, Wilding G, et al. Immediate versus deferred androgen deprivation treatment in patients with node-positive prostate cancer after radical prostatectomy and pelvic lymphadenectomy. Lancet Oncol. 2006;7:472-9.

8. Loblaw DA, Virgo KS, Nam R, Somerfield MR, Ben-Josef E, Mendelson DS, et al. Initial hormonal management of androgen-sensitive metastatic, recurrent, or progressive prostate cancer: 2006 update of an American Society of Clinical Oncology practice guideline. J Clin Oncol. 2007;25:1596-605.

9. Wong YN, Freedland S, Egleston B, Hudes G, Schwartz JS, Armstrong K. Role of androgen deprivation therapy for node-positive prostate cancer. J Clin Oncol. 2009;27:100-5.

10. Mohler JL, Kantoff PW, Armstrong AJ, Bahnson RR, Cohen M, D'Amico AV, et al. Prostate cancer, version 2.2014. J Natl Compr Canc Netw. 2014;12:686-718.

11. Engel J, Bastian PJ, Baur H, Beer V, Chaussy C, Gschwend JE, et al. Survival benefit of radical prostatectomy in lymph node-positive patients with prostate cancer. Eur Urol. 2010;57:754-61.

12. Petros JA, Catalona WJ. Lower incidence of unsuspected lymph node metastases in 521 consecutive patients with clinically localized prostate cancer. J Urol. 1992;147:1574-5.

13. Han M, Partin AW, Pound CR, Epstein JI, Walsh PC. Long-term biochemical disease-free and cancer-specific survival following anatomic radical retropubic prostatectomy. The 15-year Johns Hopkins experience. Urol Clin North Am. 2001;28:555-65.

14. Palapattu GS, Allaf ME, Trock BJ, Epstein JI, Walsh PC. Prostate specific antigen progression in men with lymph node metastases following radical prostatectomy: results of long-term followup. J Urol. 2004;172:1860-4.

15. Chuang AY, Chang SJ, Horng CF, Tsou MH. Study of prostate cancer pathologic features in Chinese populations. Urology. 2007;69:915-20

16. Bader P, Burkhard FC, Markwalder R, Studer UE. Disease progression and survival of patients with positive lymph nodes after radical prostatectomy. Is there a chance of cure? Journal Of Urology. 2003;169:849-54.

17. Chen MK, Luo Y, Zhang H, Qiu JG, Wen XQ, Pang J, et al. Laparoscopic radical prostatectomy plus extended lymph nodes dissection for cases with non-extra node metastatic prostate cancer: 5 -year experience in a single Chinese institution. J Cancer Res Clin Oncol. 2013;139:871-8.

18. Peyromaure M, Debre B, Mao K, Zhang G, Wang Y, Sun Z, et al. Management of prostate cancer in China: a multicenter report of 6 institutions. J Urol. 2005;174:1794-7.

19. Qin XJ, Ma CG, Ye DW, Yao XD, Zhang SL, Dai B, et al. Tumor cytoreduction results in better response to androgen ablation-a preliminary report of palliative transurethral resection of the prostate in metastatic hormone sensitive prostate cancer. Urol Oncol. 2012;30:145-9.

20. Mostaghel EA, Page ST, Lin DW, Fazli L, Coleman IM, True LD, et al. Intraprostatic androgens and androgen-regulated gene expression persist after testosterone suppression: therapeutic implications for castration-resistant prostate cancer. Cancer Res. 2007;67:5033-41.

21. Qin X, Ye D. Re: Jutta Engel, Patrick J. Bastian, Helmut Baur, et al. Survival benefit of radical prostatectomy in lymph node-positive patients with prostate cancer. Eur Urol 2010;57:754-61. Eur Urol. 2010;58:e43. author reply e44.

\section{Submit your next manuscript to BioMed Central and take full advantage of:}

- Convenient online submission

- Thorough peer review

- No space constraints or color figure charges

- Immediate publication on acceptance

- Inclusion in PubMed, CAS, Scopus and Google Scholar

- Research which is freely available for redistribution 